\title{
¿Contribuye la estancia en casas de acogida al proceso de empoderamiento de las mujeres víctimas de violencia de género?
}

\author{
Does the stay in women's shelters contribute \\ to their empowerment?
}

\author{
Miriam LuCAS ARRANZ* \\ Psicóloga infantil \\ Servicios Sociales de la Mancomunidad La Maliciosa y \\ en el Punto Municipal del Observatorio de Violencia de \\ Género de La Maliciosa \\ ORCID ID: 0000-0003-0940-8638 \\ Soledad Hurtado Villanueva* \\ Educadora social \\ Centro de emergencia para violencia de género de la red \\ del Ayuntamiento de Madrid \\ DESIRÉE PAZ SANZ ${ }^{* * *}$ \\ Psicóloga \\ Centro de emergencia para violencia de género de la red \\ del Ayuntamiento de Madrid
}

doi: https://doi.org/10.20318/femeris.2022.6625

Resumen. Este artículo pretende reflexionar acerca del actual funcionamiento del sistema de protección a las víctimas de violencia de género, más concretamente respecto a los recursos residenciales de protección.

Se ha realizado un estudio cualitativo mediante entrevistas a 18 profesionales, analizando su valoración de la intervención psicosocial con mujeres en estos recursos desde el enfoque del empowerment, partiendo de la base de que la mayoría de las propuestas de intervención terapéutica con mujeres víctimas de violencia de género contempla el empoderamiento como objetivo.

El 100\% de las entrevistadas coincide en que estos recursos cumplen la función de protección, pero un $88,8 \%$ considera que la estancia en casas de acogida no empodera a las mujeres por diversos motivos. Entre los argumentos señalados están los siguientes: se trabaja desde un enfoque meramente asistencialista, la normativa estricta y el control que se ejerce desde la institución sobre las actuaciones de estas mujeres en el día a día genera dificultades y

\footnotetext{
*mlmiriamlucas@gmail.com

** shurtadovillanueva@gmail.com

*** desireepazsanz@hotmail.com
} 
M. Lucas, S. Hurtado, D. Paz Sanz $\quad$ ¿Contribuye la estancia en casas de acogida al proceso de empoderamiento...

limita su independencia y autonomía, existe una falta de intimidad derivada del propio diseño de estos recursos comunitarios, la temporalidad de la estancia, que se considera insuficiente, condiciona la intervención, y el hecho base del que partimos, es decir, que sea la mujer quien tenga que refugiarse y esconderse.

Palabras clave: violencia de género, casas de acogida, empoderamiento, burocratización de los recursos, feminismo..

Abstract. This paper tries to look into the actual functioning of the Protection System for victims of gender violence, more concretely into how women's shelters work.

Based on a qualitative approach, this research has been carried out through the analysis of experienced and qualified staff interviews: 18 professionals have been asked to evaluate the psychosocial intervention in these refugees for women victims of gender violence focusing on the empowerment perspective. This standpoint has been emphasized and considered as a main objective by most of therapeutic proposals for women who have suffered gender violence.

The $100 \%$ of interviewed people considers that residential resources for victims of gender violence provide protection and security. However, the $88.8 \%$ points out that the stay in these refugees does not contribute to their empowerment. They support this idea on different reasons: these resources are still based on an aid- oriented approach, the strict normative system and institutional control (this does not allow women to be independent and autonomous), the design of these community refugees restricts their privacy, the temporary nature of these resources defines the intervention and lastly, the fact, on the basis of the intervention, that victims have to leave their homes, and stay hidden and sheltered.

Keywords: gender violence, women's shelters, empowerment, bureaucratization of resources, feminism.

\section{Introducción}

La violencia de género es un grave problema social que se ha abordado desde distintos ámbitos. En el plano internacional destacan la Conferencia Mundial de Derechos Humanos (ONU, 1993), la Declaración sobre la Eliminación de la Violencia contra la Mujer (ONU, 1993) y la Conferencia Mundial sobre la Mujer en Beijing (ONU, 1995).

En España, la Ley Orgánica de Medidas de Protección Integral contra la Violencia de Género 1/2004 de 28 de diciembre de 2004, hace referencia por primera vez en la historia al derecho de las víctimas a la asistencia social integral y multidisciplinar. Esta Ley puede considerarse la norma que ha marcado la política criminal en este ámbito en los últimos años y se ha considerado una ley inaugural e instrumental para la concienciación del sector del Derecho con respecto a esta realidad (Copello, 2005; Rubio, 2010; Maqueda Abreu, 2010; Martínez García, 2012; Gisbert, Grifo y Martínez García, 2016). Además, establece las directrices a tener en cuenta por todas las Comunidades Autónomas.

En la Comunidad de Madrid se desarrolla en esta línea la Ley 5/2005 de 20 de Diciembre, en cuyo artículo 16 se recogen los recursos de protección y acogida para mujeres víctimas de violencia de género, clasificados de la siguiente forma:

a) Centros de Emergencia. Su objetivo es dar alojamiento inmediato y seguro a las víctimas, así como manutención y otros gastos derivados de la situación con una temporalidad máxima de dos meses. Sirven de apoyo en los primeros momentos de la toma de decisiones mientras se aporta el apoyo profesional. 
b) Centros de Acogida. Su objetivo es dispensar alojamiento seguro, manutención y otros gastos a las víctimas y menores por el tiempo necesario para llevar a cabo su recuperación, sin que pueda exceder de los 12 meses. Ofrecen un tratamiento integral de recuperación desde los ámbitos psicológicos, educativo, sociolaboral y jurídico la normalización de la unidad familiar y la superación de los efectos de la violencia.

c) Pisos Tutelados. Su objetivo es dispensar alojamiento y seguimiento psicosocial a las mujeres y menores que han finalizado el proceso de atención en Centro de Acogida y que continúan precisando apoyo en la consecución de su autonomía personal por un tiempo máximo de dieciocho meses.

La creación de refugios y casas para víctimas de violencia de género surge en la década de los 60 con un movimiento feminista cada vez más visible y activista. El movimiento de liberación de las mujeres que se inicia en esta década comienza a centrarse en una problemática considerada tradicionalmente privada, por ocurrir en la intimidad del hogar: la denominada en aquel momento violencia doméstica. Surgen así los primeros refugios, creados por militancia feminista en América y Europa, organizando pisos secretos no reglamentarios y sin ayuda gubernamental (Anderson y Zinsser, 1992, 2000; Heise, 1997; Kanuha, 1997).

No es hasta la década de los 70 cuando la violencia contra las mujeres comienza a denunciarse de forma específica como problema social debido, entre otros factores, a la creciente presencia de las mujeres en el mundo laboral. Se comienza a debatir de forma contundente la normalización de la violencia dentro de la familia como un hecho que contribuye a la sumisión de las mujeres y que las coloca en desventaja con respecto a los hombres (Jovaní y cols., 1994).

Ante la falta de elementos jurídicos y de una respuesta del Estado específica a la problemática, los grupos de mujeres fueron los que, de manera informal, desarrollaron una serie de mecanismos para atender las necesidades más urgentes de las mujeres que habían sobrevivido a la violencia machista (La Torre, 2011). Uno de esos instrumentos y, por otra parte, objeto de este estudio, fueron las casas de acogida, entendidas como un espacio físico al que las mujeres pudieran acudir en caso de tener que abandonar su hogar de manera urgente.

Los refugios y casas de acogida surgen en el contexto europeo en Gran Bretaña en 1971, creándose posteriormente en otras ciudades europeas (Giebeler, 2006). Es en este año (1971) en el que Erin Pizzey organiza un grupo de mujeres y crea el primer centro comunitario de mujeres, niños y niñas en Chiswick. Este refugio se ideó como modelo abierto, en el que la dirección y los teléfonos eran públicos; a su vez, prestaba atención a las particularidades de las víctimas de la violencia de género al igual que a los agresores, con los que se esforzaba en trabajar.

La existencia de una perspectiva feminista y el sentimiento de hermandad, fueron características básicas de las primeras casas de acogida, europeas y americanas.

Sin embargo, no pasó mucho tiempo hasta que se evidenciaron las grandes dificultades existentes para su financiación, lo que derivó en la búsqueda de nuevas fuentes de ingresos, 
entrelazándose así el servicio privado y la burocracia gubernamental (Chang, 1992).

Con la intromisión y la necesidad de financiación por parte del Estado, cambia el funcionamiento de los refugios y casas de acogidas; el autocuidado y la ayuda mutua, se diluyen en una estructura piramidal. Los recursos residenciales para mujeres víctimas de violencia de género pasan a ser dirigidos por profesionales cualificadas, lo cual marca y determina el tipo de relaciones que se establecen, son guiados ahora por estructuras predominantes de poder. Confluyen los intereses de la administración y el activismo feminista, para dar una respuesta eficaz y a largo plazo a una problemática emergente, que no iba a poder sostenerse con las milicias, grupos de mujeres o financiación individual y privada (Osborne, 2008).

Con esta necesaria participación del Estado en la gestión de las casas de acogida, surge la necesidad de aportar los resultados de los procesos realizados con las supervivientes dentro del espacio del recurso, con el objetivo de hacer perdurar la financiación (Donnelly, Cook, Wilson, 1999). Acontece así un cambio de contexto y cultura, en el que se establecen unas normas de comportamiento y un derecho de admisión cada vez más restringido, además de primar una fuerte burocratización de los recursos del propio centro (Koyama, 2003).

El caso de España es sensiblemente distinto al resto de países cuyos grupos feministas comenzaron a crear pisos clandestinos, pues hasta 1975 España estaba inmersa en una dictadura en la que el Código Civil mantenía la potestad dentro del matrimonio que permitía al marido corregir a su esposa y la obligaba a obedecerlo (Larrauri, 1994). En 1983, el primer gobierno socialista de la democracia crea el Instituto de la Mujer, junto a las voces provenientes del movimiento feminista. Este hecho jugó un papel relevante en la sensibilización y en la presión social para que la violencia de género entrase en la agenda política española (Valiente, 2006).

Sin embargo, tal y como argumentaremos a lo largo del escrito, desde que confluyen los intereses del Estado dentro de la gestión y funcionamiento de las casas de acogida, dicha tarea ha estado y está, actualmente, determinada por un criterio institucional que no siempre concuerda con aquellas premisas de libertad y autonomía que guían las demandas feministas de apoyo a las mujeres que viven esta situación de violencia de género.

A diferencia de los primeros refugios, con la financiación estatal, se comenzaron a ver las casas de acogida como un servicio, a las víctimas como usuarias (o beneficiarias) y al personal como empleadas (ya no voluntarias) (Epstein, Russell, Silvern, 1998). A medida que el número de refugios crecía, se iban haciendo más responsables con los requisitos de los financiadores, provocando un aumento de las reglas internas relacionadas con los criterios de acceso, la confidencialidad de ubicación, el contacto con las personas del entorno, los quehaceres domésticos, toques de queda, crianza de los hijos e hijas y abuso de sustancias (Chang, 1992; Goodman \& Epstein, 2008; Rodriguez, 1988; Haaken y Yrugai, 2003). Así comienza a establecerse una fuerte legislación interna sobre quién puede acceder, quién puede hacer qué, cuándo y cómo (Samuelson, Wilson, 2004).

La confidencialidad es una de las normas más importante de estos recursos. Las mujeres tienen la prohibición explícita de indicar la dirección y zona de la casa de acogida a cual- 
quier persona: familiar, pareja actual, instituciones, redes de apoyo social, etc. Durante su estancia en el refugio, se indicarán unas "zonas de peligro", a las que la mujer no podrá acudir por ningún motivo y que, generalmente, incluyen las zonas de sus redes sociales, familiares e institucionales creadas, probablemente, a lo largo de un amplio periodo de tiempo. Por supuesto, y siguiendo esta norma, las mujeres tienen prohibido el contacto con sus agresores ya que esto puede vulnerar la seguridad de todas las residentes (Schillinger, 1988).

Janice Haaken y Nan Yragui (2003) plantean que el hecho de mantener el secreto de la localización del centro supone tensiones extras que también obstaculizan la intervención.

En este sentido cabe destacar el caso de Finlandia, pues, si bien los países de Europa entienden, por regla general, los refugios y casas de acogida como sistemas cerrados y protegidos, Finlandia toma ejemplo del primer refugio abierto en Inglaterra por Erin Pizzey. Opta por una red de refugios abiertos, con direcciones y teléfonos públicos, en los que el trabajo se realiza de forma individualizada con víctimas y victimarios y, en ocasiones, también de forma conjunta, dando mucha importancia a la prevención de la violencia trabajada desde la rehabilitación del agresor (Ericksson et al., 2005).

La normativa de los recursos limita también el tiempo de ausencia de los mismos, es decir, se exponen unos horarios de estancia obligatorios (comidas, toques de queda, hora de dormir) y, por otro lado, se dan limitaciones de espacio, donde se establecen las áreas para comer, fumar, recreación, almacenamiento de comida y de medicamentos (con limitado o inexistente acceso a ellos). Estas reglas, también pueden requerir que las mujeres asistan a un número determinado de grupos de apoyo, talleres y sesiones de intervención (Holden, 1997; Schillinger, 1988).

Lisa Goodman y Deborah Epstein (2008), describen cuatro consecuencias principales de la asociación del movimiento feminista con el Estado con respecto a los refugios. Estos incluyen: estructuras de organización jerárquicas y prevalencia de pirámides de poder; mayor grado de profesionalización del personal que trabaja en los recursos; mayor atención a la eficiencia y a los resultados para dar continuidad a la financiación; criterios de entrada más estrictos.

Así pues, los refugios han desarrollado un creciente número de criterios de selección, siendo el resultado de las regulaciones estatales (Chang, 1992). No pueden ingresar mujeres con diagnóstico psiquiátrico o con consumo abusivo de sustancias y/o alcohol, siendo el argumento para ello, la convivencia de la residencia (Sáez Ulloa, 2017).

Esta presión sistemática sobre los refugios, obligó a desarrollar estructuras de poder convencionales y a contratar personal tradicionalmente acreditado con roles claramente delimitados (Dobash \& Dobash, 1987). Las diferencias entre profesionales y residentes se vuelven más estrictas, restringiendo el espíritu igualitario y el sentido de comunidad tan aferrado que existía en los primeros refugios, incluso, prohibiendo explícitamente la "confraternización" con las residentes (Chang, 1992).

Tal y como expresa Hobart (2006) la forma en la que se comunican las normas y se hacen cumplir, son un símbolo de cultura de poder y crea una relación jerárquica entre las personas que trabajan en las casas de acogida y las propias mujeres, pudiendo relacionarse con roles de madre/hija, rescatadora/víctima o maestro/estudiante. 
Una de las cuestiones más discutidas en torno al establecimiento de reglas tan estrictas en las casas de acogida, supone la poca autocrítica de las mismas por parte de los ejecutores de las normas (profesionales). Algunos autores como Denise Donnely, Kimberly Cook y Linda Wilson (1999) señalan que el personal de los refugios, abrumado por la necesidad de aportar efectividad por escrito, crea reglas en medio del caos de la vida convivencial en el refugio y, luego, jamás se vuelven a cuestionar dichas normas.

Se pierde así la esencia del movimiento feminista comunitario, que parte de la base de la intervención social, considerando que la desigualdad en el reparto de los recursos materiales y psicológicos provoca problemas sociales. (Zimmerman, 2000).

La psicología comunitaria tiene como objetivo promover unas condiciones de vida saludables, objetivo que promueve a partir del modelo del empowerment (Hombrados, 1996; Musitu y Buelga, 2004).

Tener que presentar datos exitosos y una justificación continua del trabajo, define las reglas existentes en los refugios con respecto al diseño de la intervención en lugar de permitir que cada mujer defina por sí misma lo que precisa y necesita de su estancia en la casa (Smyth, Goodman y Glenn, 2006).

En la mayoría de los casos, las mujeres beneficiarias del recurso de acogida tendrán que cambiar de barrio, de escuela, de amistades, deberán interrumpir las relaciones familiares cercanas, las amistades que tengan en común con los agresores adaptándose así a las reglas de la casa (La Torre, Roig, 2011).

En cuanto al rol de madre, desde los recursos de acogida, también viene determinado por la intervención profesional, pues el personal de los refugios se involucra en la crianza de los menores residentes, realizando talleres para madres, prohibiciones como dejar a los niños y niñas sin supervisión y establecimiento de horarios educativos (Cosgrove \& Flynn, 2005; Holden, 1997). Estas reglas de intromisión en la crianza tienden a generar un sentimiento de degradación, impotencia y control (Haj Yahia \& Cohen, 2009), entendiendo que las madres pueden sentir cuestionada su autoridad y su papel maternal.

La presencia de las profesionales en las casas de acogida es un factor que tiene mucho peso en el día a día y en cómo las mujeres viven esta experiencia. Las profesionales juegan un papel ambivalente; por un lado, tienen la función de acompañar a las mujeres durante todo su proceso y, por otro, tienen la función de hacer cumplir las normas existentes. Las víctimas pueden vivir su estancia como una pérdida de poder de decisión sobre su propia vida, y pueden visualizar cómo el plano tan íntimo como es el de la personalidad está siendo afectado al pasar por el recurso (La Torre, Roig, 2011).

Por otra parte, a lo largo de su estancia, las residentes tendrán la obligatoriedad de involucrarse en la búsqueda de empleo y vivienda, sabiendo en todo momento que su tiempo de estancia en la casa de acogida es limitado y que, en caso de incumplir la normativa impuesta, podrían tener consecuencias negativas que acarreen una pronta salida del refugio (Haj Yahia \& Cohen, 2009).

Teniendo en cuenta lo descrito y la conversión que han sufrido los refugios a lo largo de las décadas desde su creación, la cuestión que nos atañe es si este sistema institucionalizado y reglamentario es capaz de dar solución real a las necesidades de las víctimas. 
¿Realmente es capaz un sistema normativo estricto de otorgar confianza, autonomía y empoderamiento a las víctimas de violencia que acuden a él? ¿Se puede desarrollar la toma de decisiones y la identidad propia real desde un sistema como el descrito?

En 1977, Julian Rappaport incluye el modelo de empowerment, partiendo de la contribución de los recursos individuales, grupales y comunitarios como potenciadores del bienestar y calidad de vida (Buelga, 2007).

El enfoque del empowerment no pretende resolver un problema concreto sino desarrollar las potencialidades del individuo, grupo o sistema social para aumentar el bienestar y calidad de vida (Montero, 2003).

En 1993, Srilatha Batliwala explica que el empoderamiento está relacionado con el control sobre los recursos, materiales y humanos, y el control sobre la ideología, es decir, creencias, valores y actitudes. Cuando un proceso empieza a crear conciencia sobre la persona, pero no otorga un mayor control sobre los recursos suele conllevar el abandono. El empoderamiento pretende reajustar las relaciones del individuo con su entorno, la falta de libertad y de poder que afectan negativamente a la salud y bienestar (Kar, Pascual y Chickering, 1999). No consiste meramente en el acceso a los recursos, sino del control (Kishor, 1999; Za Sathar y Kazi, 1997) y la capacidad de elección sobre ellos (Alsop y Heinsohn, 2005).

En cuanto a la relación del empoderamiento y la vulnerabilidad ante una situación de violencia de género, Sunita Kishor (2000), Zoe Oxaal y Sally Baden (1997) establecen los siguientes elementos del empoderamiento femenino como factores de protección frente a la violencia en el ámbito de la pareja: autonomía entendida como libertad de movimiento y acción, poder de decisión, educación formal, participación en las luchas colectivas, generación de ingresos.

Otras autoras también señalan el empoderamiento como elemento fundamental en los procesos de recuperación de violencia de género:

Isabel Rebollo y Cristina Bravo (2005), en esta línea, destacan que todas las áreas que participan en el proceso de intervención deben promover la autonomía personal y la responsabilidad, favorecer la toma de decisiones y proporcionar estrategias de afrontamiento para el futuro que sean capaces de prevenir violencias machistas.

Ma Pilar Matud y cols. (2004) han elaborado un programa de tratamiento integral basado en el desarrollo de las potencialidades con el objetivo final de empoderar a las mujeres para la recuperación del control sobre sus propias vidas.

Por su lado, Leonor Walker (1996) desarrolla "Survivor Therapy" integrando estrategias de intervención de la terapia del trauma con los principios de la teoría feminista y, también, pone énfasis en la necesidad de trabajar el empoderamiento, además de la seguridad, validación de su experiencia, refuerzo de sus capacidades, comprensión de la opresión y toma de decisiones.

Victoria A. Ferrer Pérez y Aina Alzamora (2005) subrayan así mismo el empoderamiento como uno de los puntos de su programa de tratamiento para mujeres víctimas de violencia de género.

No obstante, estos problemas relativos al trabajo en casas de acogida ya han sido previamente analizados desde diferentes perspectivas y desde distintos países. 
En un estudio realizado en Chile se describieron dificultades encontradas en la intervención en casas de acogida (Sáez Ulloa, 2017). Señala este estudio, en primer lugar, algunos aspectos estructurales, como la injusticia percibida en el hecho de que sean ellas quienes tengan que ingresar en un centro y no los agresores, que en muchos casos permanecen en libertad. Por otro lado, menciona los problemas de coordinación con instituciones que participan del proceso de intervención de las mujeres y que desconocen el operar de la residencia, dificultando el avance administrativo. Desde la perspectiva de la intervención contextual destaca el concepto de temporalidad como punto clave de la intervención. Las orientaciones técnicas declaran que el tiempo de permanencia de las mujeres en estas casas de acogida es entre tres y seis meses. La experiencia y la práctica, por el contrario, apuntan a que este tiempo es insuficiente para la generación de procesos terapéuticos, la recuperación y el establecimiento de redes de apoyo sólidas. Además, constatan que este conflicto entre los objetivos y la temporalidad invisibiliza en muchos casos, las necesidades reales de las mujeres.

Tal y como subraya esta autora:

"Se declara que se han dado situaciones en las cuales miembros del equipo, por cumplir con las metas y exigencias institucionales, ejercen presión sobre los procesos de intervención con las usuarias, recalcándoles el carácter transitorio de la residencia y que desde el ingreso se debe pensar en el medio para su salida. Situación que si bien se reconoce es necesario tener presente, no siempre es considerado como algo positivo para los procesos reparatorios". (Sáez Ulloa, 2017)

Expone al respecto, que, a juicio de los equipos técnicos consultados, desde las propias instituciones se mantienen prácticas culturales que reproducen formas de violencia y que vulneran los derechos de la mujer, ya que la normativa y los protocolos de actuación en ocasiones se imponen a su voluntad. Soledad Calle (2004) remarca que, teniendo en cuenta que las relaciones agresor-víctima están basadas en el dominio y en la sumisión, la relación asistencial no debería reproducir un sistema jerárquico.

Marchant Espinoza (2014), en este sentido, apunta a que las dificultades que las mujeres experimentan en las casas de acogida para adaptarse a la estricta normativa impuesta, generan en ellas sentimientos de angustia obstaculizando la superación del evento traumático.

A pesar de que numerosos estudios hacen referencia a problemas de estructuración del sistema, tal y como se ha expuesto, muchos otros ponen el foco en la actuación de las profesionales que trabajan en estas casas de acogida; actuación que está inevitablemente relacionada con el sistema y la estructura sociopolítica que da respuesta a esta problemática.

Soledad Calle (2004) afirma que el proceso denuncia -separación- casa de acogida resulta ineficaz en la mayoría de los casos y que, de hecho, la mayoría de las mujeres rechaza ingresar en este tipo de recursos, percibiéndolos como una sanción para ellas. 
Se han revisado algunas de las complicaciones descritas en la literatura relativas al funcionamiento de los equipos. El trabajo en una casa de acogida es complejo por diversas razones: la sobrecarga emocional derivada de las propias situaciones de violencia experimentadas por las usuarias, la multiplicidad de asuntos que atender dentro y fuera de la casa (convivencia y coordinación con otros recursos), o, como apuntaba Giannina Sáez Ulloa, el doble rol del equipo (por un lado, hacer cumplir las normas y, por otro lado, contribuir en el proceso terapéutico). La obligatoriedad del cumplimiento de dichas normas puede devaluar el proceso de intervención, llevando en el peor de los casos a adoptar desde el ámbito profesional un rol coercitivo y controlador, alejándose de la relación de ayuda y la empatía (Lucas Arranz, 2015).

En esta misma línea, un equipo de profesionales de la casa de acogida de Castellón detectó que el 50\% de las mujeres atendidas no alcanzaba los objetivos planteados, de modo que hicieron cambios analizando sus propias actitudes. Destacaron la importancia de atender a la especificidad de cada caso, algo que dificulta las estadísticas, pero que resulta esencial para que el tratamiento sea efectivo, permitiendo a las mujeres ser agentes activos en su propio proceso; subrayaron la importancia del respeto por la diferencia por encima del prejuicio y en el peligro del saber sobreentendido. Ponen énfasis estas autoras en la necesidad de supervisión emocional de los equipos, reciclaje y formación continuada para analizar la subjetividad que afecta a las profesionales (Grupo Cala, 1994). La ausencia de espacios reflexivos, tanto para establecer criterios y pautas de actuación comunes (Rebollo y Bravo, 2005), o enfocados al estado emocional en el que se encuentran individualmente las trabajadoras (Sáez Ulloa, 2017), también ha sido señalada como obstáculo del proceso de intervención por otros equipos profesionales.

Algunos riesgos para las profesionales que trabajan en este ámbito serían el desgaste por empatía o la falacia de cambio. La falacia de cambio es descrita por Isabel Rebollo y Cristina Bravo (2005) como la creencia de poder cambiar a otras personas, lo que nos llevaría a exigir a otras personas que realicen cambios acordes a nuestra opinión. En el caso del trabajo con mujeres víctimas de violencia es frecuente que las profesionales experimenten sentimientos de tristeza y frustración cuando las mujeres regresan o toman contacto con los agresores. Esta implicación emocional por parte de las profesionales es un elemento muy negativo para el proceso de recuperación delas víctimas.

Estas mismas autoras resaltan la necesidad de la interseccionalidad a la hora de plantearse el trabajo desde estos centros. Las mujeres que ingresan en estos recursos, por lo general, vienen rodeadas de una compleja multiproblemática (Lucas Arranz, 2015). Isabel Repollo y Cristina Bravo (2005) analizaron los perfiles atendidos en una casa de acogida de la Comunidad de Madrid durante 2 años, concluyendo que un $80 \%$ eran mujeres inmigrantes, el 50\% de los casos presentaba otros problemas además de haber sido víctimas de violencia de género, y un 25\% presentaban psicopatologías que no habían sido tratadas ni diagnosticadas. Subrayan las dificultades para encontrar recursos que respondan de manera global a las necesidades encontradas.

Erin Pizzey remarca la vulnerabilidad que se produce en las mujeres que además están en situación de pobreza. Los perfiles atendidos en estos centros son sumamente 
vulnerables y la forma en que se establecen los límites y normas debe ser cuidadosamente analizada. En la negociación de estos límites, se ha puesto atención a la interdependencia del racismo, sexismo y opresión de clase en la perpetuación de los ciclos de abuso (Hamby, 2000; Mechanic, 1996; Renzetti, 1994; Uzzell and Peebles-Wilkins, 1989; West, 1998).

No obstante, la consideración de dichos factores, género, raza y clase socio-económica, debe ir más allá de buscar un mero traductor para casos en que el idioma sea diferente (Haaken y Yragui, 2003). Un ejemplo de esto lo constituiría la idea de amor romántico en las diferentes culturas. ¿Viven de la misma forma el amor en China que en España? No se puede trabajar por igual la separación o la ruptura familiar desde una óptica musulmana que latinoamericana, ni ignorar la idiosincrasia de cada mujer.

Tal y como Kimberlé Crenshaw (1994) señala, las prácticas elitistas que se realizan de manera rígida en muchos centros vienen por parte de feministas blancas. En Reino Unido, Erin Pizzey criticó las políticas del Estado de Bienestar Social y la respuesta que se daba a las problemáticas de familias en situación de pobreza, considerándola punitiva e insuficiente. Desde las teorías sociológicas y médicas, se ponía el foco en buscar la psicopatología de dichas familias. Erin Pizzey fue, de hecho, criticada por otras feministas británicas por alienarse con los roles de poder a nivel profesional (psicología, trabajo social), que buscaban tratamiento más que políticas sociales.

Muchas mujeres de las que acuden a estos recursos, como ya hemos apuntado, presentan problemas económicos además de otras problemáticas psicosociales de diversa índole, por lo que la intervención no debería limitarse a abordar exclusivamente la violencia de género experimentada, sino también las múltiples formas de dominación que a menudo convergen en ellas (Crenshaw, 1994: 95).

Janice Haaken y Nan Yragui (2003) recogen la idea de que los centros feministas deberían organizarse bajo principios democráticos y no jerárquicos, trabajando de manera colectiva y política para cambiar las condiciones en que viven las mujeres. Este funcionamiento facilitaría el empoderamiento de estas mujeres y contribuiría a su participación como agentes activos en su proceso de cambio. Sin embargo, la rigidez operativa de la mayoría de estos recursos dificulta la relación de ayuda sin roles de poder, sin barreras que posicionen a las profesionales por encima de las usuarias.

\section{Método}

Se ha realizado un estudio cualitativo, mediante entrevistas a personas con diferentes roles profesionales: psicólogas, trabajadoras sociales, educadoras sociales y auxiliares educativas, que han desempeñado su trabajo en recursos de atención a mujeres víctimas de violencia de género.

La muestra está compuesta por 18 entrevistas que constan de una pregunta central: 
¿Crees que ayuda la estancia en los centros de emergencia y/o casas de acogida en el proceso de empoderamiento de las víctimas?

De la muestra se extraen los siguientes resultados (Tabla 1):

Tabla 1. Características de las personas entrevistadas

\begin{tabular}{|c|c|c|}
\hline Categoría profesional & Años de experiencia & $\begin{array}{c}\text { ¿Empodera la estancia } \\
\text { en casas de acogida? }\end{array}$ \\
\hline Psicóloga 1 & Más de 10 & NO \\
\hline Psicóloga 2 & Más de 10 & NO \\
\hline Psicóloga 3 & Más de 10 & NO \\
\hline Psicóloga 4 & Más de 10 & NO \\
\hline Psicóloga 5 & Más de 10 & NO \\
\hline Psicóloga 6 & De 2 a 5 años & SI \\
\hline Psicóloga 7 & Más de 10 & NO \\
\hline Psicóloga 8 & Más de 10 & NO \\
\hline Psicóloga 9 & Más de 5 & NO \\
\hline Trabajadora social 1 & Más de 10 & NO \\
\hline Trabajadora social 2 & Más de 10 & NO \\
\hline Educadora 1 & Más de 10 & NO \\
\hline Educadora 2 & Más de 10 & NO \\
\hline Auxiliar 1 & Menos de 2 años & SI \\
\hline Auxiliar 2 & Menos de 2 años & SI \\
\hline Auxiliar 3 & De 2 a 5 años & NO \\
\hline Auxiliar 4 & De 2 a 5 años & NO \\
\hline Auxiliar 5 & De 2 a 5 años & NO \\
\hline
\end{tabular}

En la tabla 1 se clasifican en una respuesta cerrada (Si-No) las valoraciones de las diferentes profesionales entrevistadas acerca del empoderamiento en las casas de acogida, especificando en cada caso variables como la categoría profesional y años de experiencia en el sector.

La siguiente tabla resume y agrupa las principales razones a las que se han atribuido las dificultades mencionadas por las profesionales entrevistadas para trabajar el empoderamiento desde las casas de acogida (Tabla 2):

Tabla 2. Argumentos expuestos en las entrevistas

\begin{tabular}{|l|c|}
\hline Argumento expuesto & $\begin{array}{c}\text { Personas que } \\
\text { lo mencionan }\end{array}$ \\
\hline Las casas de acogida aportan protección. & 18 \\
\hline $\begin{array}{l}\text { Se presta apoyo jurídico y burocrático, lo que contribuye al proceso de } \\
\text { empoderamiento. }\end{array}$ & 1 \\
\hline Se ofrece ayuda para gestiones administrativas que ayudan a su independencia. & 1 \\
\hline Existe un enfoque meramente asistencialista que no ayuda al empoderamiento. & 5 \\
\hline La rigidez de la normativa y funcionamiento institucional impiden el empoderamiento. & 16 \\
\hline
\end{tabular}




\begin{tabular}{|l|c|}
\hline Argumento expuesto & $\begin{array}{c}\text { Personas que } \\
\text { lo mencionan }\end{array}$ \\
\hline La temporalidad de la estancia es un obstáculo. & 10 \\
\hline $\begin{array}{l}\text { El funcionamiento institucional genera dependencia y dificultad posterior para la toma } \\
\text { de decisiones. }\end{array}$ & 7 \\
\hline $\begin{array}{l}\text { El diseño de los pisos genera falta de independencia y es un obstáculo para el } \\
\text { empoderamiento. }\end{array}$ & 3 \\
\hline $\begin{array}{l}\text { El propio sistema de protección evita el empoderamiento, haciendo que la mujer } \\
\text { tenga que "esconderse" renunciando a demasiados aspectos de su vida y de su } \\
\text { independencia. }\end{array}$ & 5 \\
\hline
\end{tabular}

De las respuestas abiertas recopiladas en las entrevistas se extraen los argumentos principales y se agrupan contabilizando el número de personas que hacen alusión a cada uno.

Argumentos positivos

Argumentos negativos

\section{Análisis de resultados}

Un 83,34\% de las entrevistadas ha respondido que, en su opinión, las casas de acogida no contribuyen al empoderamiento de las mujeres residentes, frente a un $16,66 \%$ que considera que sí.

El 100\% de profesionales con más de 10 años de experiencia considera que la estancia en casas de acogida no empodera.

Las únicas respuestas que han considerado lo contrario (un 16,66\%) han sido dadas por personas con menor experiencia laboral en el sector $(11,1 \%$ menos de dos años y el $5,5 \%$ menos de cinco).

Esta discrepancia podría deberse a varios motivos:

Por un lado, podría ocurrir que las profesionales con más experiencia, por el síndrome de burn-out, fueran más críticas o tuvieran una visión más negativa o distorsionada del funcionamiento institucional.

Otra hipótesis sería que las profesionales con menor experiencia no hubieran tenido tiempo de analizar bien los efectos de la estancia en las casas de acogida, o que la motivación por iniciarse en el mundo laboral provoque un sesgo en la percepción.

Teniendo en cuenta que existen personas con menos experiencia que también considera que dicha estancia no contribuye al proceso de empoderamiento de las mujeres (un 16,66\%) cobra más peso la segunda hipótesis.

No obstante, hay que recordar que las profesionales estarán condicionadas por su propia forma de hacer, sus ideas y valores así como su propio estilo educativo, lo que generará de por sí discrepancias en el análisis de estos procesos terapéuticos.

Se exponen a continuación las ideas principales extraídas de las distintas entrevistas realizadas.

Respecto a las razones ofrecidas por las diferentes profesionales, cabe destacar que el 100\% coincide en que se aporta protección a las víctimas. Así mismo, el 11,1\% señala que el acompañamiento para los trámites jurídicos y administrativos contribuye a su proceso de independencia y por lo tanto, de forma indirecta a su empoderamiento. 
M. Lucas, S. Hurtado, D. Paz Sanz $\quad$ ¿Contribuye la estancia en casas de acogida al proceso de empoderamiento...

A pesar de subrayar la labor de protección que cumplen estos recursos, un 38.88\% señala que es precisamente esta sobreprotección la que infantiliza a las mujeres, genera dependencia institucional, dificultad para la toma de decisiones, y ausencia de perspectiva realista sobre un proyecto de vida autónomo a corto, medio plazo.

Por otro lado, un $27,7 \%$ de las entrevistadas destaca la idea de que existe una evidente falta de coherencia en el discurso actual de los centros de acogida, remarcando el carácter asistencialista de los mismos. A continuación se reproduce textualmente un fragmento de una de las entrevistas que ilustra esta idea:

"Por un lado, encontramos un discurso igualitario, feminista y potenciador de habilidades mientras que, en la práctica, nos topamos con una mera prestación de servicios y una forma de organización poco democrática, que se basa en hacer cumplir unas reglas inamovibles, que no han sido consensuadas con las víctimas y que, ni las entidades privadas que, en muchos casos, gestionan estos centros, ni el Estado, se han preocupado en replantear, cuestionar ni estudiar de forma sistemática con el paso del tiempo, considerándolas apropiadas y útiles sin tener en cuenta los perfiles ni grupos de mujeres en cada momento".

Un $88,8 \%$ señala la normativa como principal obstáculo para el empoderamiento. Aparecen las siguientes ideas al respecto:

Durante su estancia en los centros, muchas veces las mujeres se limitan a cumplir rígidamente unas normas institucionales, normas que las aíslan (al condicionar por completo su vida social) y tutelan. Queda condicionada así toda la intervención terapéutica por un miedo a posibles amonestaciones derivadas del incumplimiento de reglas que en muchos casos ni siquiera comprenden, y la amenaza, siempre latente, de resultar expulsadas del recurso. Esto puede generar un efecto totalmente contrario al empoderamiento; una reducción de la autonomía, de su toma de decisiones, desánimo, frustración y sentimiento de pérdida de control sobre sus propias vidas.

"Muchas mujeres refieren sentir el mismo control y autoridad que experimentaban en la relación de violencia, aunque en realidad no sea asi" plantea una de las entrevistadas.

Así pues, esta sensación de control puede llegar a distorsionar la relación de ayuda, visualizando a las profesionales como enemigas en vez de aliadas en su proceso de recuperación.

Se señalan dos características de las casas de acogida como obstáculos: el diseño de las mismas y la temporalidad.

Un 16, 6\% considera que el propio diseño de las casas (pisos compartidos por varias mujeres o unidades familiares que en ocasiones deben compartir incluso habitación) dificulta la autonomía y toma de decisiones respecto a sus propias vidas.

Por otro lado, un 55,5\% de las entrevistadas subraya la temporalidad como barrera terapéutica para el empoderamiento. Algunas realizan una distinción entre centro de emergencia (destinado a un primer momento de crisis) y casa de acogida (que haría referencia a una estancia de mayor duración), argumentando que en los primeros resulta más 
complejo el trabajo del empoderamiento al estar la intervención definida por su carácter de urgencia. Sin embargo, la percepción y valoración de la estancia por parte de la usuaria en ese primer momento será determinante en el desarrollo del proceso de intervención, por lo que independientemente de las características temporales de los recursos, la base y la direccionalidad de la intervención hacia procesos de autonomía y empoderamiento deberían establecerse desde el inicio.

En esta línea, atendiendo a la base de los procesos de intervención, en un 27,7\% de las entrevistas ha aparecido la idea de que el hecho de que la mujer tenga que abandonar el hogar y "esconderse", genera la sensación de que el sistema castiga a la propia mujer, la revictimiza alejándola de su red social, obligándola a cambiar de domicilio, trabajo, colegio de los menores..., aislándola, sometiéndola a un sistema normativo estricto en aras de su seguridad. El poder y control quedan en manos de terceros, como ocurría en su relación.

\section{Conclusiones}

A pesar de que actualmente se da respuesta a las necesidades asistenciales y de seguridad vital de las mujeres que sufren violencia de género a manos de sus parejas o exparejas a través de una red de casas de acogida, según entrevistas realizadas a profesionales con experiencia en este sector, el funcionamiento institucional de estos recursos no contribuye al empoderamiento y autonomía de la mujer; un 88,8\% considera esta idea, frente al 16,66\% que afirma que la estancia en casas de acogida sí ayuda al empoderamiento de las mujeres.

Las dificultades señaladas son las siguientes: existe un enfoque meramente asistencialista, la rigidez de la normativa de las casas impide el empoderamiento, la intervención está definida por la temporalidad y el diseño de los centro, las características de la intervención psicosocial en este ámbito generan dependencia institucional y por último, el hecho de que el propio sistema de protección "castiga" a la víctima obligándola a permanecer escondida en estas casas de acogida.

Por otro lado, el $100 \%$ de las entrevistadas coincide en que las casas de acogida cumplen la función de protección.

Así pues, aunque las profesionales son cada vez más cualificadas, el funcionamiento jerárquico de los propios recursos y la progresiva burocratización de los mismos, limitan la labor profesional perdiendo de vista algunos pilares básicos de la intervención psicosocial y del feminismo como es el empoderamiento como forma de liberación y progreso.

\section{Bibliografía}

ALSOP, RUTH and HEINSOHN, NINA. Measuring Empowerment in Practice: Structuring Analysis and Framing Indicators (February 2005). Available at SSRN: https://ssrn. com/abstract $=665062$ 
M. Lucas, S. Hurtado, D. Paz Sanz $\quad$ ¿Contribuye la estancia en casas de acogida al proceso de empoderamiento...

ANDERSON, BONNIE y ZINSSER, JUDITH (1991). Historia de las mujeres. Una historia propia. Crítica: Barcelona.

ANDERSON, BONNIES y ZINSSER, JUDITH (1999). A history of their own. Volume II. Women in Europe from prehistory to the present. Oxford University Press. New York.

BATLIWALA, S. Asian-South Pacific Bureau of Adult Education., \& Freedom from Hunger Campaign, Action for Development. (1993). Empowerment of women in South Asia: Concepts and practices. New Delhi, India: FFHC/AD Programme Officer.

BOSCH FIOL, ESPERANZA, FERRER-PÉREZ, VICTORIA A. y ALZAMORA MIR, AINA (2005). Algunas claves para una psicoterapia de orientación feminista en mujeres que han padecido violencia de género. Feminismo/s, 6, 121-136.

BUELGA, SOFÍA (2007). El "empowerment" la potenciación del bienestar desde la psicología comunitaria. En M. Gil (Dir), Psicología social y bienestar: una aproximación multidisciplinar (pp. 154-173). Universidad de Zaragoza.

CHANG, DEANNA BAU KUNG (1992). A domestic violence shelter: A symbol of bureaucracy. Social Process in Hawaii, 34, pp 37-52.

CHAVEZ PLAZAS, YURI y MARCHANTE ESPINOZA, JUAN PAULO (2014). Nuevas masculinidades en desplazamiento: construcciones sociales y culturales del significado de ser hombre. Una mirada desde Chile y Colombia. Tabula Rasa, 21, pp 287-303.

COHEN, HILLA CHAYA y HAJ-YAHIA, MUHAMMAD M. (2009). On the Lived Experience of Battered Women Residing in Shelters. Journal of Family Violence 24(2), pp. 95-109.

COSGROVE, LISA y FLYNN, CHERYL (2005). Marginalized mothers: Parenting without a home. Analyses of Social Issues and Public Policy, 5, pp 127-143.

CRENSHAW KIMBERLÉ WILLIAMS (1994). Mapping the Margins: Intersectionality, Identity Politics, and Violence Against Women of Color, pp. 93-118 en Albertson Fineman, Martha y Mykitiuk, Rixanne, The Public Nature of Private Violence: The Discovery of Domestic Abuse. New York: Routledge

DOBASH, R. EMERSON y DOBASH, RUSELL P. (1987). The response of the British and American women's movement to violence against women, en Hanmer, J. y Maynard, M. (eds), Women, violence social control (pp. 169-179). London: Macmillan Press.

DONNELLY, DENISE A., COOK, KIMBERLY J. y WILSON, LINDA A. (1999) Provision and exclusion: the dual face of services to bettered women in three Deep South States. Violence Agains Women 6 (7), 710-741.

EPSTEIN, SARA, RUSSELL, GLENDA y SILVERN, LOUISE (1988). Ideology and organizational age as influences on the development of battered women's shelters. American Journal of Community Psychology, 16, 345-367.

GIEBELER, CORNELIA (2006). Engendering Social Work: Conceptos teóricos y metodológicos feministas con el trabajo social. Acciones e investigaciones sociales, 1, 123.

GISBERT GRIFO, SUSANA y MARTÍNEZ GARCÍA, ELENA (2016). Género y violencia. Valencia: Tirant lo Blanch.

GOODMAN, LISA A. y EPSTEIN, DEBORAH (2008). Listening to battered women: a survivorcentered approach to advocacy, mental health, and justice. Washington, D.C.: American Psychological Association. 
M. Lucas, S. Hurtado, D. Paz Sanz $\quad$ ¿Contribuye la estancia en casas de acogida al proceso de empoderamiento...

HAAKEN, JANICE y YRAGUI, NAN (2003). Going underground: Conflicting perspectives on domestic violence shelter practices. Feminism \& Psychology, 13, 49-71.

HAMBY, SHERRY L. (2000). The Importance of Community in a Feminist Analysis of Domestic Violence Among American Indians. American Journal of Community Psychology 28, 649-69.

HEISE, LORI L (1997). La violencia contra la mujer. Organización global para el cambio, en J. Edleson y Z. Eisikovits. Violencia doméstica. La mujer golpeada y la familia (pp. 1958). Buenos Aires: Granica.

HOBART, MARGARET (2006). Changing the script: Thinking about our relationships with shelter residents. Recuperado de http://www.wscadv.org/resourcesPublications.cfm

HOLDEN, DAPHNE (1997). On equal ground: Sustaining virtue among volunteers in a homeless shelter. Journal of Contemporary Ethnography, 26, 117-145.

HOMBRADOS, MARÍA ISABEL (1996). Introducción a la psicología comunitaria. España: Aljibe.

JOVANÍ, ESTHER, MARTÍ, PILAR, SEGARRA, TERESA y TORMO, M.J. (1994). Mujeres maltratadas, mujeres mal tratadas. Asparkía 4, 9-18.

KANUHA, VAL KALEI (1997). Violencia doméstica, racismo y el movimiento de mujeres golpeadas en Estados Unidos. En Edleson, J y Eisikovits, Z. Violencia doméstica. La mujer golpeada y la familia (pp. 59-82). Buenos Aires: Granica.

KAR, SNEHENDU B., PASCUAL, CATHERINE A. y CHICKERING, KRISTIN L. (1999). Empowerment of women for health promotion: A meta-analysis. Social Science \& Medicine, 49(11), 1431-1460. Recuperado de https://doi.org/10.1016/S0277-9536(99)00200-2

KISHOR, SUNITA (2000). Women's Contraceptive Use in Egypt: What do Direct Measures of Empowerment Tell Us? Annual meeting of the Population Association of America, 23-25. Los Angeles, California.

KISHOR, SUNITA y NEITZEL, KATHERINE (1996). The Status of Women: Indicators for Twenty-Five Countries. Demographic and health Surveys Comparative Studies, 21. Calverton, Maryland USA: Macro International.

KOYAMA, EMI (2003). Disloyal to feminism: Abuse of survivors within the domestic violence shelter system. Recuperado de http://www.confluere.com/store/pdf-zn/disloyal2.pdf LARRAURI, ELENA (1994) Mujeres, derecho penal y criminología. Madrid: Siglo XXI.

LA TORRE, ELENA y ROIG, AURA (2001) Los recursos de acogida temporal para mujeres sobrevivientes a situaciones de violencia machista intrafamiliar. Crítica penal y poder: una publicación del Observatorio del Sistema Penal y los Derechos Humanos, 1 pp 88-116.

LAURENZO COPELLO, PATRICIA (2005) La violencia de género en la Ley integral. Valoración políticocriminal, RECPC 07-08, p. 1.

Ley Orgánica 1/2004 de 28 de diciembre de Medidas de Protección Integral contra la Violencia de Género, Boletín Oficial del Estado, número 313 de 29 de diciembre de 2004, pp 42.166- 42.197. Recuperado de https://www.boe.es/boe/dias/2004/12/29/ pdfs/A42166-42197.pdf 
M. Lucas, S. Hurtado, D. Paz Sanz $\quad$ ¿Contribuye la estancia en casas de acogida al proceso de empoderamiento...

Ley 5/2005 de 20 de diciembre, integral contra la violencia de género de la Comunidad de Madrid. Boletín Oficial del Estado, número 52 de 2 de marzo de 2006, pp 8515-8526. Recuperado de https://www.boe.es/buscar/doc.php?id=BOE-A-2006-3667

LUCAS ARRANZ, MIRIAM (2015). Una propuesta de intervención para niños y niñas en edad preescolar expuestos a violencia de género. En Sofía Czalbowski (coord.). Detrás de la pared (pp 287-313). Madrid: Desclée

MAQUEDA ABREU, MARÍA LUISA (2010). 1989-2009: Veinte años de "desencuentros" entre la ley penal y la realidad de la violencia en la pareja, en Laurenzo Copello, Patricia. (2010). La violencia de género en la Ley. Reflexiones sobre veinte años de experiencia en España. Madrid: Dykinson.

MARTÍNEZ GARCÍA, E. (2012). Reflexiones tras seis años de aplicación de la Ley, en Martínez García, E. La prevención y la erradicación de la Violencia de Género. Un estudio multidisciplinar y forense (p.577). Cizur menor: Thomson Reuters-Aranzadi.

MATUD, M. PILAR (2004). Diseño y validación de un programa de intervención psicológica con mujeres víctimas de maltrato por parte de su pareja. Informe de investigación. Instituto de la Mujer, Madrid.

MECHANIC, MINDY B. (1996). Battered Women, Homicide, and the Legal System. en Roberts, A.R. Helping Battered Women: New Perspectives and Remedies. (pp. 132-56). New York: Oxford University Press.

MUSITU, GONZALO y BUELGA, SONIA (2004). Desarrollo Comunitario y potenciación. En Musitu, G., Herrero J., Cantera, Leonor, y Montenegro Marisela. Introducción a la Psicología Comunitaria. (pp. 167-192). Barcelona: Barcelona UOC.

MONTERO, MARITZA (2003) Teoría y práctica de la psicología comunitaria: la tensión entre comunidad y sociedad. Buenos Aires, Paidós.

ORGANIZACIÓN DE LAS NACIONES UNIDAS (1993). Declaración sobre la Eliminación de la violencia contra la Mujer. Recuperado de https://www.ohchr.org/SP/ProfessionaIInterest/Pages/ViolenceAgainstWomen.aspx

ORGANIZACIÓN DE LAS NACIONES UNIDAS (1995). Conferencia Mundial sobre la Mujer en Beijing. Recuperado de https://www.un.org/womenwatch/daw/beijing/pdf/ Beijing\%20full\%20report\%20S.pdf

OSBORNE, RAQUEL (2008). De la «violencia» (de género) a las "cifras de la violencia": una cuestión política. Empiria. Revista de metodología de ciencias sociales, 15.doi: https://doi.org/10.5944/empiria.15.2008.

OXAAL, ZOE y BADEN, SALLY (1997). Gender and Empowerment: definitions, approaches and implications for policy. Brighton, UK: Bridge, Institute of Development Studies.

REBOLLO SÁNCHEZ, ISABEL y BRAVO, CRISTINA (2005). Casas de acogida: desde la experiencia a la reflexión. Cuadernos De Trabajo Social, 18, 317-332.

RUBIO, ANA (2010) La Ley integral: entre el desconcierto del género y la eficacia impuesta, en Laurenzo Copello, Patricia. La violencia de género en la Ley. Reflexiones sobre veinte años de experiencia en España (p.131). Madrid: Dykinson.

RENZETTI, CLAIRE M. (1994). On Dancing with the Bear: Reflections on Current Debates Among Domestic Violence Theorists, Violence and Victims 9(2), 195-200. 
M. Lucas, S. Hurtado, D. Paz Sanz $\quad$ ¿Contribuye la estancia en casas de acogida al proceso de empoderamiento...

SAMUELSON, CHARLES D. y WILSON, RICK K. (2004). Investigating conflict, power, and status within and among groups. Small Group Research, 35, 44-72.

SÁEZ ULLOA, GIANNINA (2017). Intervención social en violencia grave hacia la mujer: Distinciones de las profesionales de un programa Casas de Acogida de SERNAM (Tesis). Recuperado de http://repositorio.uchile.cl/handle/2250/16784

SATHAR, ZEBA AYESCA y KAZI, SHAHNAZ (1997). Women's Autonomy, Livelihood \& Fertility: A Study of Rural Punjab. Islamabad: Instituto Pakistan Institute of Development Economics.

SCHILLINGER, ELISABETH (1988). Dependency, control, and isolation: Battered women and the welfare system. Journal of Contemporary Ethnography, 16, 469-490.

SMYTH, KATYA., GOODMAN, LISA A. y GLENN, CATHERINE (2006). The Full-Frame Approach: A new response to marginalized women left behind by specialized services. American Journal of Orthopsychiatry 76(4), 489-502.

UZZELL, ODELL y PEEBLES-WILKINS, WILMA (1989). Black Spouse Abuse: A Focus on Relational Factors and Intervention Strategies. The Western Journal of Black Studies 13(1): 10-16.

VALIENTE, CELIA (2006). El feminismo de estado en España: el Instituto de la Mujer (19832003). Valencia: Institut d'Estudis Universitaris de la Dona.

WEST, TRACI C. (1999). Wounds of the Spirit: Black Women, Violence, and Resistance Ethics. New York: New York University Press.

ZIMMERMAN, BARRY J. (2000). Attaining self-regulation. A social cognitive perspectives en M. Boekaerts, M., Pintrich, P.R. y Zeidner, M. (eds.), Handbook of self-regulation, (pp. 13-39). London: UK Academic Press. 